\title{
BRAQUITERAPIA DE ALTA TAXA DE DOSE NO BRASIL*
}

\author{
Sérgio Carlos Barros Esteves ${ }^{1}$, Antonio Carlos Zuliani de Oliveira ${ }^{2}$, Luís Fernando \\ de Andrade Feijó ${ }^{2}$
}

Resumo A braquiterapia de alta taxa de dose foi introduzida em nosso meio em janeiro de 1991. Desde então, houve uma mudança significativa na abordagem das neoplasias malignas em relação às vantagens do novo método, e também resolução da demanda reprimida de braquiterapia para as neoplasias ginecológicas. Nos primeiros dez anos de atividade, o Brasil tratou, em 31 serviços, 26.436 pacientes com braquiterapia, sendo mais de $50 \%$ das pacientes portadoras de neoplasias do colo uterino. Este estudo mostra o número e o perfil de pacientes tratados com esse método e a sua distribuição no território nacional, deixando explícito o benefício da braquiterapia de alta taxa de dose para o Brasil.

Unitermos: Braquiterapia de alta taxa de dose; Câncer; Neoplasia.

Abstract High-dose rate brachytherapy in Brazil.

High-dose rate brachytherapy was first introduced in Brazil in January 1991. Significant changes in the management of malignant neoplasms were observed since utilization of high-dose rate brachytherapy. The high number of gynecological patients awaiting for brachytherapy also decreased during this period. In the first ten years 26,436 patients were treated with high-dose rate brachytherapy. More than $50 \%$ of these patients presented neoplasms of the uterine cervix. In this study we present the number and profile of the patients treated with high-dose rate brachytherapy as well as the distribution of these patients in the Brazilian territory, proving the benefit of the use of high-dose rate brachytherapy in Brazil.

Key words: High-dose rate brachytherapy; Cancer; Neoplasms.

\section{INTRODUÇÃO}

\section{Definição e histórico}

Em janeiro de 2001, a radioterapia nacional completou dez anos de experiência com braquiterapia de alta taxa de dose (BATD). A braquiterapia é uma modalidade terapêutica da radioterapia em que se utilizam fontes radioativas em íntimo contato com a região a ser tratada. O objetivo deste tratamento é administrar altas doses de radiação em volumes restritos do organismo, para se ter maior controle da doença e menor toxicidade do tratamento aos tecidos normais adjacentes.

O conceito de BATD não é recente. No início do século passado, apesar de não se falar em dose e sim em miligrama.hora (mg.h) para braquiterapia, o sistema de Estocolmo tinha a filosofia de tratar com

* Trabalho realizado no Centro de Assistência Integral à Saúde da Mulher (CAISM) da Universidade Estadual de Campinas (Unicamp), Campinas, SP.

1. Doutor, Chefe da Seção de Radioterapia do CAISM-Unicamp, Radio-oncologista do Hospital Beneficência Portuguesa de São Paulo.

2. Médicos Radioterapeutas do CAISM-Unicamp.

Endereço para correspondência: Dr. Sérgio Esteves. Departamento de Radioterapia, CAISM-Unicamp. Rua Alexander Fleming 101, Cidade Universitária Zeferino Vaz. Campinas, SP, 13083 970. E-mail: estevesrt@uol.com.br

Recebido para publicação em 22/8/2003. Aceito, após revisão, em 17/10/2003. grande quantidade de material radioativo (alta atividade), em curto espaço de tempo. A BATD é definida como o tratamento cuja taxa de dose é superior a 0,2 Gy/min (ou $12 \mathrm{~Gy} / \mathrm{h}$ ). A média taxa ficaria entre 2 e $12 \mathrm{~Gy} / \mathrm{h}$ e a baixa taxa de dose seria de 0,4 a $2 \mathrm{~Gy} / \mathrm{h}^{(\mathbf{1})}$.

Na década de 60, Henschke foi o introdutor da braquiterapia com fontes de cobalto por meio de controle remoto. As fontes em miniatura para o tratamento com BATD foram introduzidas na década de 80 . Desde então, aumentou consideravelmente a versatilidade da radioterapia para o tratamento de lesões em situações clínicas o mais variado possível. Pode-se aplicar a braquiterapia para tratar desde cavidades minúsculas ou órgãos oco-musculares, até interstícios com lesão presente ou mesmo com risco de recidiva, por meio de agulhamentos e cateteres plásticos.

Todas essas facilidades de implantes, associadas ao sistema de planejamento computadorizado com transporte da carga por controle remoto, fizeram deste método o responsável pelo grande avanço da braquiterapia.

A radioterapia brasileira tem número reduzido de serviços em relação às suas necessidades, principalmente nas regiões
Norte e Nordeste, onde a incidência dos tumores do colo uterino figura em primeiro lugar entre os tumores femininos ${ }^{(2)}$.

No final da década de 80 , muitas pacientes portadoras de neoplasia do colo uterino não eram tratadas com braquiterapia. Naquele momento, fazia-se apenas braquiterapia de baixa taxa de dose (BBTD). Por características próprias desse tratamento, necessidade de internação e tempo de tratamento, muitas pacientes eram tratadas apenas com teleterapia (radiação externa), que comprometia, de maneira significativa, o resultado do tratamento ${ }^{(3)}$.

No Brasil, a BATD foi incorporada à prática clínica em janeiro de 1991, quando se instalou o primeiro equipamento de BATD da América Latina, localizado no Serviço de Radioterapia do Hospital das Clínicas da Faculdade de Medicina da Universidade de São Paulo. Tratava-se de um momento crítico, pois alguns trabalhos publicados neste período alertavam para os riscos de complicações da $\mathrm{BATD}^{(4)}$, tendo em vista a curta experiência com esta nova modalidade terapêutica ${ }^{(5)}$. Em virtude dos conceitos de radiobiologia até aquele momento, acreditava-se que os tecidos normais de resposta tardia poderiam sofrer sérios danos em longo prazo. 
Considerações sobre o efeito biológico

A radiobiologia estuda os diferentes efeitos físicos, químicos e biológicos da irradiação dos tecidos com radiações ionizantes. O efeito biológico depende de vários fatores relacionados ao tipo de radiação utilizada, à forma de administração, ao fracionamento, à taxa de dose, às condições do meio como oxigenação e pH, e ao tipo de tecido que absorve a radiação, entre outros.

No volume tratado com BATD, além do tumor, vários tecidos recebem diferentes doses com taxas de dose específicas, dependendo da sua distância da fonte radioativa. Esses tecidos respondem ao tratamento de maneira própria.

Como a experiência anterior era com BBTD, tentou-se encontrar a dose/fracionamento de BATD que correspondesse ao tratamento clássico com BBTD.

Dale et al. ${ }^{(6,7)}$ estudaram esta correlação através da fórmula do modelo linearquadrático, que não é o modelo ideal, mas é o que mais se aproxima da realidade. Dentro das limitações existentes, estabeleceu-se que, para um tratamento padrão (sistema de Manchester, dose de 60 Gy, 0,8 Gy/h no ponto A em 72 horas), o número de frações de BATD deveria ficar em torno de $17^{(\mathbf{8})}$. Neste caso, não foi levada em consideração a diferença de reparo entre tecido normal e tumoral. Considerando-se uma maior taxa de reparo para o tecido tumoral, o número de frações poderia ser de seis a sete.

Orton $^{(9)}$ definiu "janela terapêutica" como sendo o intervalo entre as curvas de controle tumoral $(75 \%)$ e complicações $(5 \%)$. Estudou diferentes esquemas de tratamento de braquiterapia, desde baixa taxa até diferentes doses de alta taxa. Notou que, com o aumento da taxa de dose e da dose por fração, a "janela terapêutica" se estreita e também aumenta o risco de complicações (Gráficos 1 e 2).

A experiência mundial não correspondeu, na prática, aos prognósticos teóricos feitos para a BATD. O próprio Orton ${ }^{(10)}$, em 2001, publicou novo artigo justificando os bons resultados com BATD em relação ao controle e à toxicidade do método. Se considerado o tempo de reparo do tecido de resposta tardia em torno de 1,5 h, o efeito biológico da BATD acabaria superando a BBTD.

Havia, inicialmente, algumas dificuldades técnicas em relação ao domínio dos aplicadores, do sistema de planejamento e do melhor fracionamento de dose. Iniciaram-se os procedimentos com muita cautela, diante das incertezas da literatura. Por outro lado, alguns trabalhos mostravam resultados excelentes de BATD no tratamento das neoplasias do colo uterino ${ }^{(\mathbf{1 1})}$, tanto em relação ao controle local da doença e à sobrevida global quanto à toxicidade do método. O tempo gasto para se realizar o procedimento, que girava em torno de 90 minutos, rapidamente caiu pela metade. Ano após ano, os serviços do Brasil foram se equipando com BATD e, aos poucos, conquistaram experiências com resultados muito satisfatórios.

O procedimento de BATD, em especial para as neoplasias do colo uterino, resolveu um sério problema da radioterapia nacional. Sendo, no Brasil, o tumor do colo uterino o mais freqüente entre as mulheres no início da década de 90, pôdese tratar grande quantidade de pacientes ambulatorialmente e com qualidade.

O objetivo deste trabalho é situar a BATD brasileira em seus primeiros dez anos de existência, enfocando os seguintes aspectos:

1. Número total de equipamentos instalados no período de janeiro de 1991 a julho de 2001.

2. Distribuição destes aparelhos dentro do território nacional.

3. Número de pacientes tratados até o momento e distribuição das neoplasias mais freqüentes.

4. Protocolos em andamento.

\section{MATERIAIS E MÉTODOS}

Os levantamentos foram realizados via fax, telefone e e-mail dos serviços cadastrados junto às empresas que comercializam equipamentos de BATD. Estes serviços responderam a um questionário especificamente elaborado para este estudo (Quadro 1).

\section{RESULTADOS}

Todas as instituições entrevistadas responderam ao questionário.

O Brasil dispunha, até julho de 2001, de 31 equipamentos de BATD instalados e distribuídos de acordo com o descrito na Tabela 1.

No período de janeiro de 1991 a julho de 2001 foram tratados 26.436 pacientes,

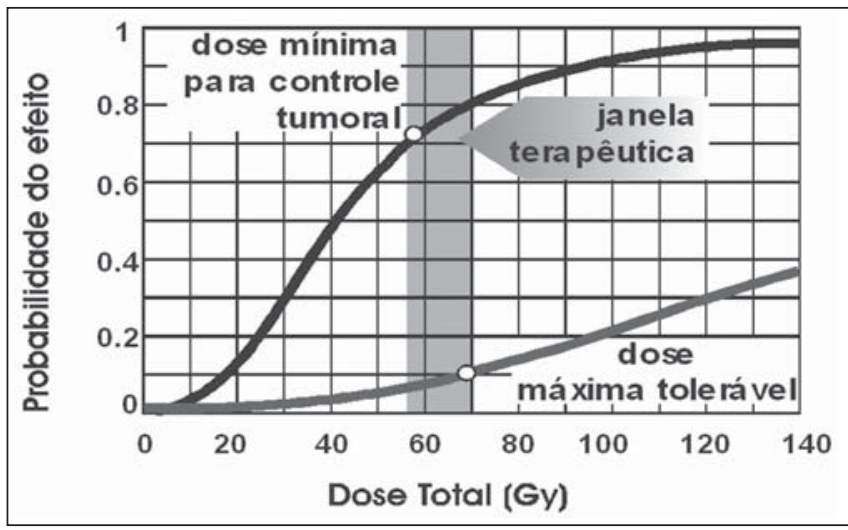

Gráfico 1. Curva de dose/resposta para taxa de dose de 1,0 Gy/h. Janela terapêutica grande.

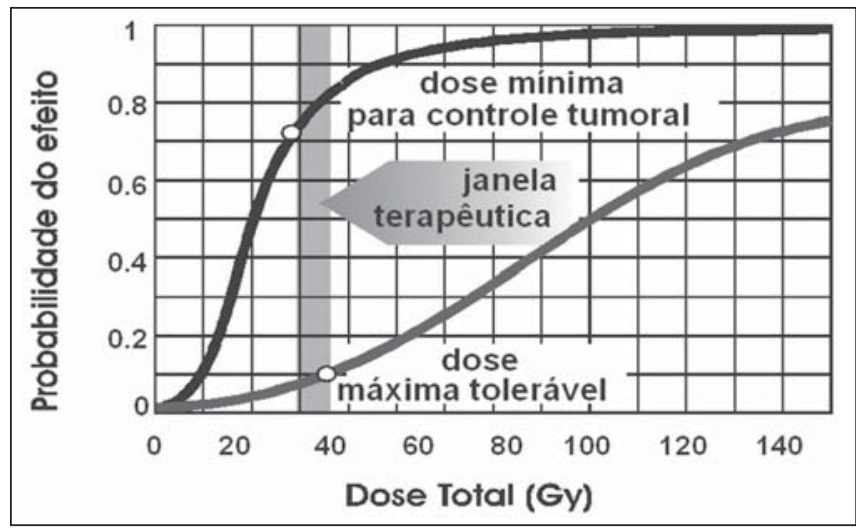

Gráfico 2. Curva de dose/resposta para taxa de dose de 12 Gy/h. Janela terapêutica pequena. 
Quadro 1.

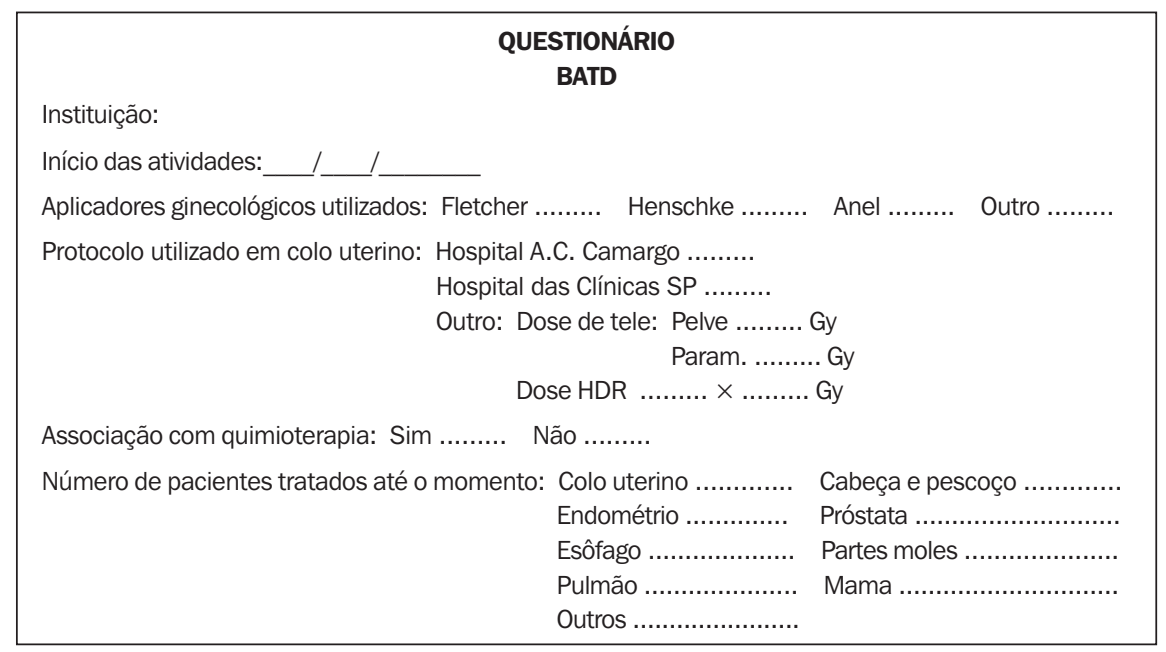

Tabela 1 Distribuição dos aparelhos de BATD pelo Brasil.

\begin{tabular}{|l|c|}
\hline \multicolumn{1}{|c|}{ Estado } & Número de aparelhos \\
\hline São Paulo & 13 \\
Rio de Janeiro & 3 \\
Minas Gerais & 2 \\
Amazonas & 2 \\
Ceará & 2 \\
Rio Grande do Sul & 2 \\
Goiás & 1 \\
Distrito Federal & 1 \\
Mato Grosso & 1 \\
Rondônia & 1 \\
Pernambuco & 1 \\
Paraíba & 1 \\
Bahia & 1 \\
\hline
\end{tabular}

a maioria portadora de tumores ginecológicos, seguidos de tumores da próstata. A distribuição, de acordo com as neoplasias mais freqüentes, está mostrada na Figura 1.

Os Gráficos de 3 a 11 mostram as incidências das neoplasias tratadas nos diferentes serviços, entre 1991 e 2001.

Os protocolos de tratamento utilizados em todos os centros são muito parecidos no que diz respeito à dose total e fracionamentos para o tratamento dos tumores ginecológicos. As doses de teleterapia ficam ao redor de 39,6 a $50 \mathrm{~Gy}$, com complementação de paramétrios variando de 9 a 20 Gy. A fração de braquiterapia varia de 6 a 7,5 Gy, num total de quatro frações. Para a braquiterapia de cúpula vaginal, a dose varia de 4 a 7 Gy, calculados de 0 a 5 $\mathrm{mm}$ da superfície do aplicador.

Entre os aplicadores, o de "anel" é o mais freqüentemente utilizado em nosso meio ${ }^{(12)}$, seguido pelo aplicador de Fletcher $^{(11)}$, Henschke ${ }^{(3)}$ e associação de aplicadores $^{(\mathbf{6})}$ (Figura 2).

Questionadas sobre o uso de quimioterapia associada ao tratamento ionizante, 12 $(38,7 \%)$ das instituições responderam não, $11(35,4 \%)$ responderam sim, seis $(19,3 \%)$ responderam eventualmente e dois $(6,4 \%)$ não responderam (Figura 3).

\section{DISCUSSÃO}

Certamente, o Brasil se destaca como um dos países com maior experiência, se não o maior, no tratamento dos tumores do colo uterino com BATD.

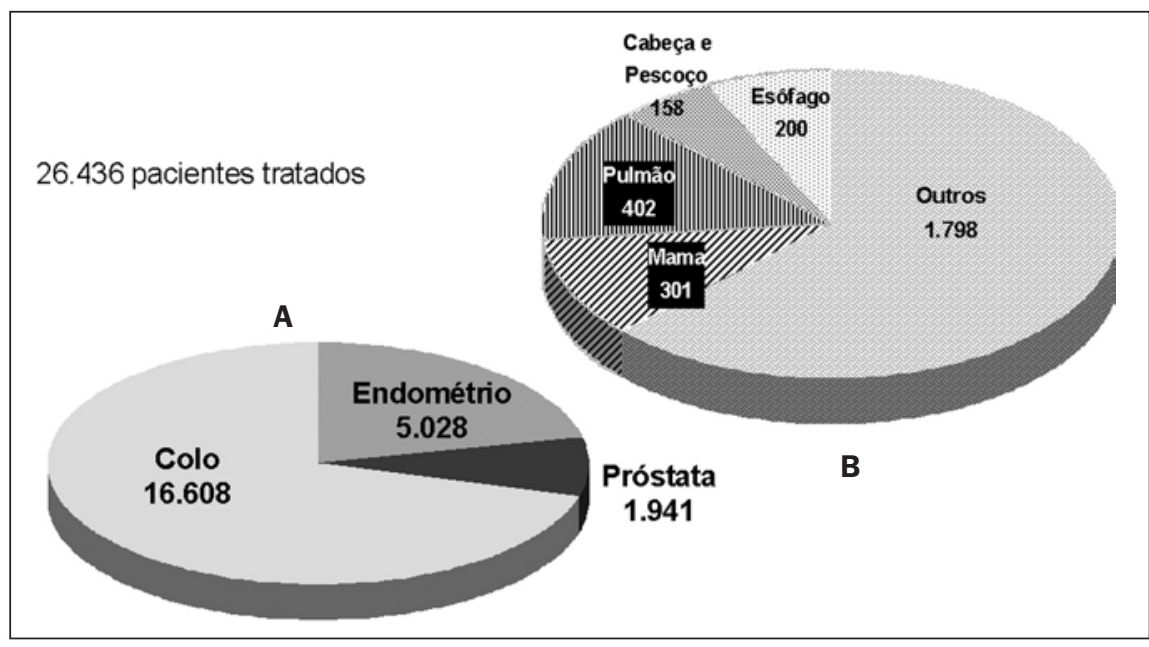

Figura 1. A: Distribuição dos pacientes portadores de neoplasia de colo, endométrio e próstata: 23.577 (89\%). B: Distribuição dos pacientes portadores de neoplasia de esôfago, cabeça e pescoço, pulmão, mama e outros: 2.859 (11\%).

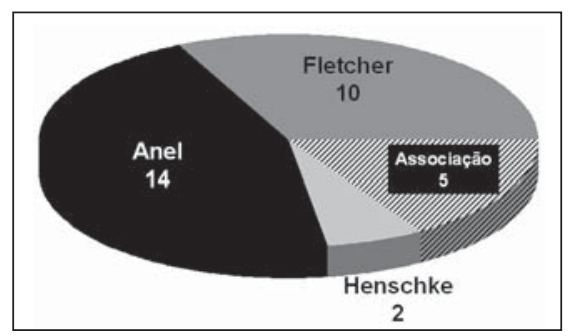

Figura 2. Distribuição dos tipos de aplicadores ginecológicos utilizados nos procedimentos de braquiterapia.

O melhor caminho para os diagnósticos precoces de tumores do colo uterino está nos programas de prevenção. Não obstante, muitos diagnósticos ainda são feitos em estádios avançados da doença. Para essas pacientes, resta o tratamento com radiote-

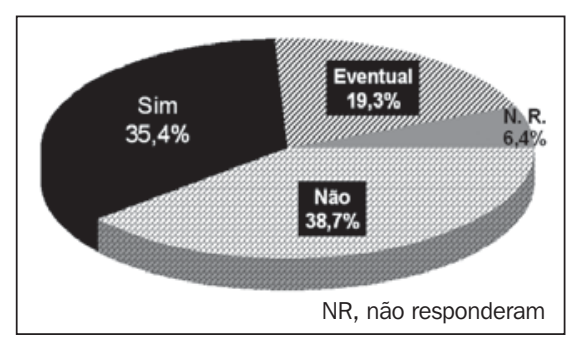

Figura 3. Proporção de seviços que utilizam radioterapia associada à quimioterapia no tratamento dos tumores do colo uterino.

rapia, que, nos dias atuais, pode proporcionar maior conforto ao paciente e maior segurança aos profissionais envolvidos.

Os serviços nacionais com mais de cinco anos de atuação, como o Hospital das Clínicas de São Paulo e o Hospital A.C. 


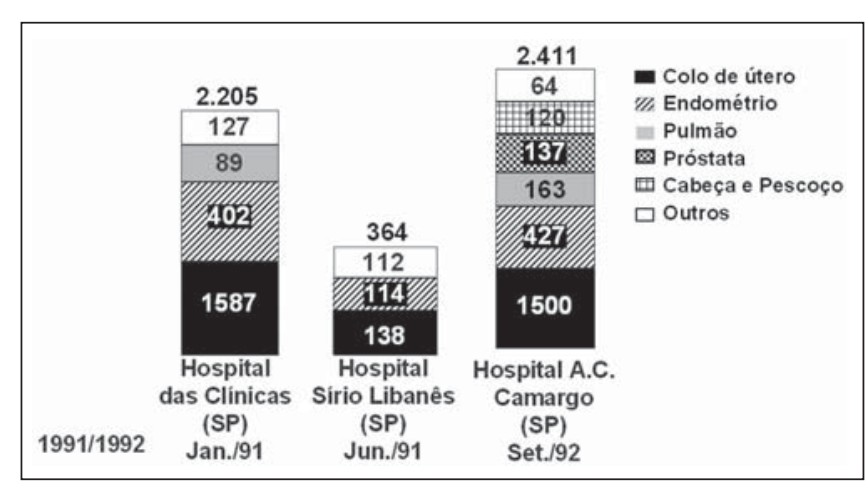

Gráfico 3. Número de pacientes tratados e distribuição das neoplasias em seus respectivos serviços (inaugurados em 1991/1992).

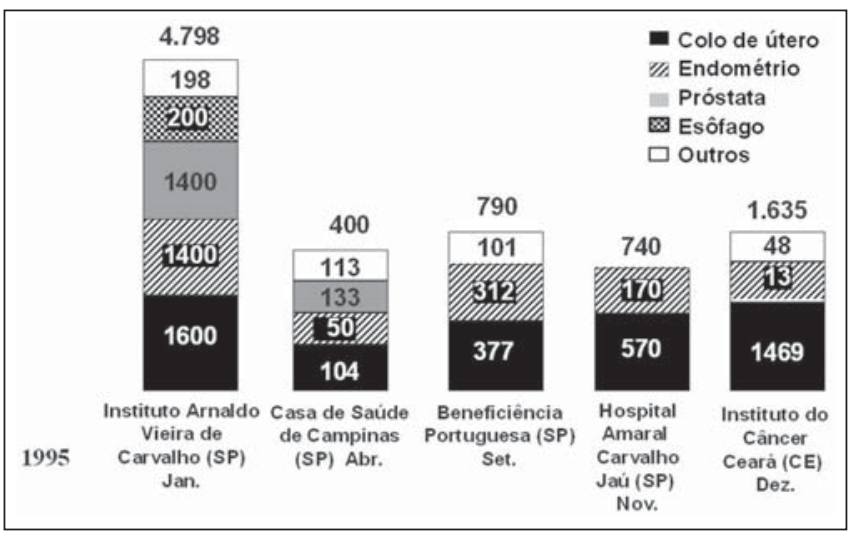

Gráfico 5. Número de pacientes tratados e distribuição das neoplasias em seus respectivos serviços (inaugurados em 1995).

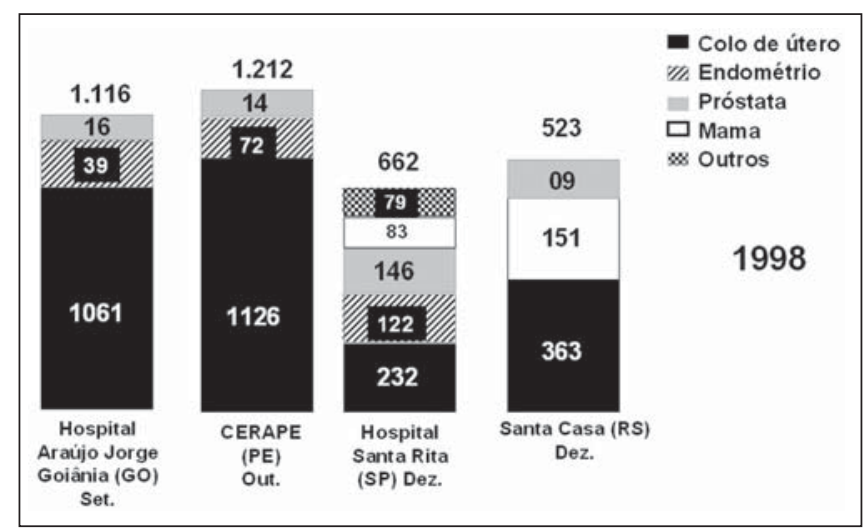

Gráfico 7. Número de pacientes tratados e distribuição das neoplasias em seus respectivos serviços (inaugurados em 1998).

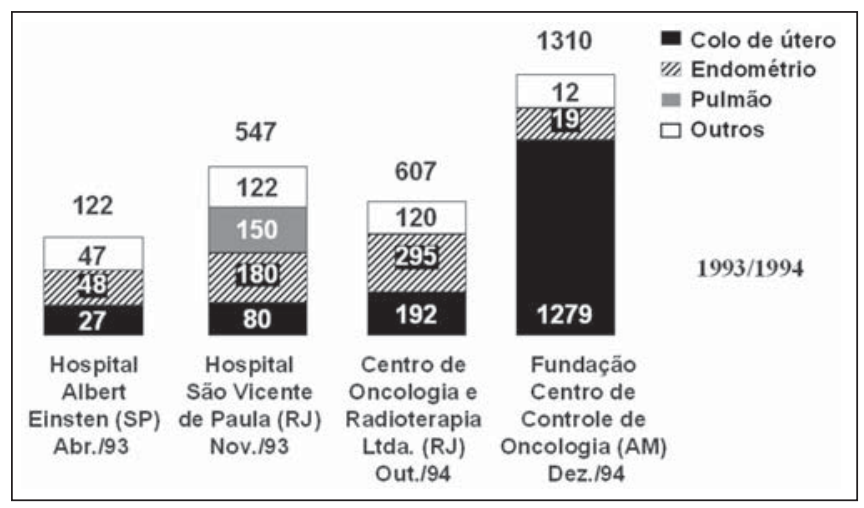

Gráfico 4. Número de pacientes tratados e distribuição das neoplasias em seus respectivos serviços (inaugurados em 1993/1994).

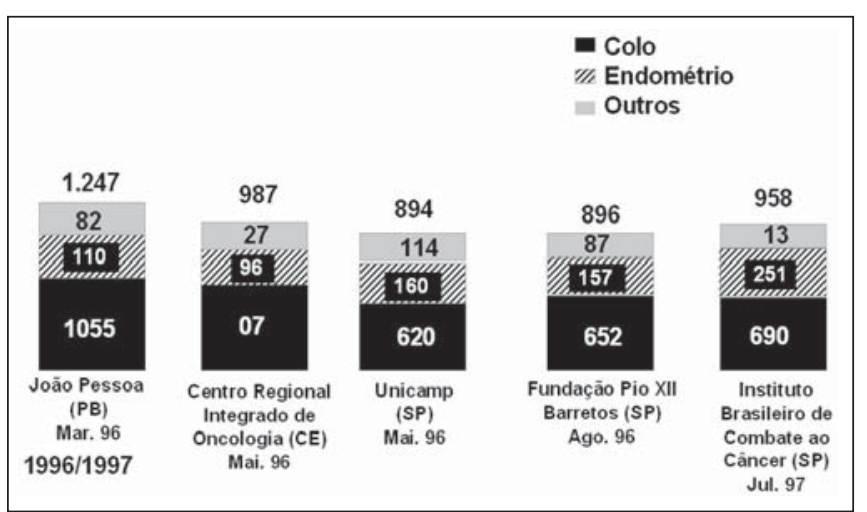

Gráfico 6. Número de pacientes tratados e distribuição das neoplasias em seus respectivos serviços (inaugurados em 1996/1997).

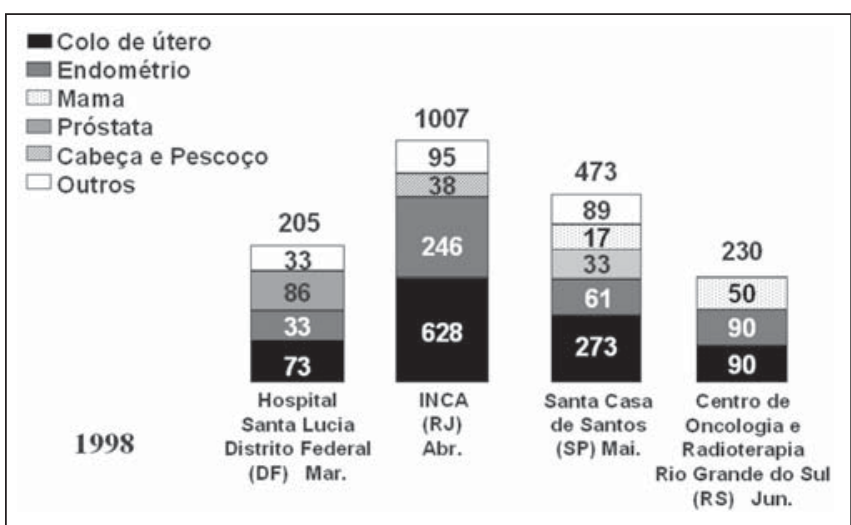

Gráfico 8. Número de pacientes tratados e distribuição das neoplasias em seus respectivos serviços (inaugurados em 1998).
Camargo, têm mostrado resultados comparáveis aos melhores já publicados na literatura $^{(\mathbf{1 3}-15)}$. Dessa forma, tratam-se os pacientes com maior segurança.

A associação da BATD com quimioterapia para tumores do colo uterino é ainda experimental. É preciso maior experiência antes de torná-la procedimento padrão.
Fica evidente a grande aplicabilidade da BATD quando se nota o número de pacientes tratados por outras neoplasias, além dos tumores ginecológicos.

Os tumores de próstata são os tumores mais freqüentes entre os homens no Brasil, depois dos tumores de pele não-melanomas, e a braquiterapia é bem indicada para pacientes selecionados. Considerando-se a região anatômica da próstata e a proximidade com os órgãos pélvicos, a braquiterapia permite menor toxicidade $\mathrm{e}$ doses mais elevadas na próstata ${ }^{(\mathbf{1 2})}$

Os tumores de pulmão são os responsáveis pelos maiores índices de mortalidade, proporcionalmente à sua incidên- 


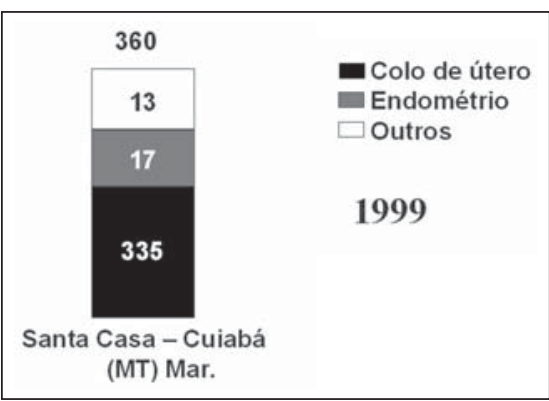

Gráfico 9. Número de pacientes tratados e distribuição das neoplasias em seus respectivos serviços (inaugurados em 1999).

cia $^{(2)}$. Freqüentemente nos deparamos com lesões endobrônquicas que causam obstrução ou sangramentos. Nestas situações, a BATD tem importante papel com finalidade paliativa ${ }^{(\mathbf{1 6})}$. Quando as lesões ainda são iniciais, pode-se ainda obter a cura da doença ${ }^{(17)}$.

Vários outros tumores, como os de cabeça e pescoço, esôfago, mama, reto, vias biliares e uretra, podem ser curados ou ter seus sintomas aliviados quando tratados com BATD. Por todos esses motivos, a radioterapia vive um momento de alta tecnologia, precisão e versatilidade.

A BATD causou grande impacto social no país. Com 26.436 pacientes tratados, pode-se afirmar que nunca foram atendidos tantos pacientes com braquiterapia como neste período de 1991 a 2001. Conclui-se que a radioterapia brasileira tornase impraticável sem a BATD, considerando-se o perfil dos pacientes do Sistema Único de Saúde. Pôde-se associar qualidade à quantidade.

\section{Agradecimentos}

Não seria possível realizar este trabalho sem o espírito colaborador de todos os profissionais dos serviços entrevistados. Nossos sinceros agradecimentos àqueles

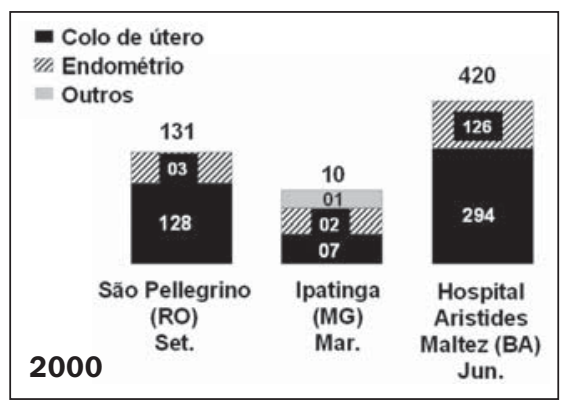

Gráfico 10. Número de pacientes tratados e distribuição das neoplasias em seus respectivos serviços (inaugurados em 2000).

que, de alguma forma, participaram do levantamento desses dados.

\section{REFERÊNCIAS}

1. International Comission on Radiation Units and Measurements. ICRU Report 38. Dose and volume specification for reporting intracavitary therapy in gynecology. Bethesda, MD: ICRU, 1985.

2. Klingerman J. Estimativas sobre incidência e mortalidade por câncer no Brasil em 2001. (Editorial). Rev Bras Cancerol 2001;47(2).

3. Coia L, Won M, Lanciano R, Marcial VA, Martz K, Hanks G. The patterns of Care Outcome Study for cancer of the uterine cervix. Results of the Second National Practice Survey. Cancer 1990;66:24516.

4. Eifel PJ. High-dose-rate brachytherapy for carcinoma of the cervix: high tech or high risk? Int J Radiat Oncol Biol Phys 1992;24:383-6.

5. Teshima T, Chatani M, Hata K, Inoue T. High-dose rate intracavitary therapy for carcinoma of the uterine cervix: II. Risk factors for rectal complication. Int J Radiat Oncol Biol Phys 1988;14:281-6.

6. Dale RG, Huczkowski J, Trott KR. Possible dose rate dependence of recovery kinetics as deduced from a preliminary analysis of the effects of fractionated irradiations at varying dose rates. $\mathrm{Br} \mathrm{J}$ Radiol 1988;61:153-7.

7. Dale RG. The use of small fraction numbers in highdose-rate gynaecological afterloading: some radiobiological considerations. Br J Radiol 1990;63: 290-4.

8. Orton CG. What minimum number of fractions is required with high-dose rate remote afterloading? Br J Radiol 1987;60:300-2.

9. Orton CG. Width of the therapeutic window: what is the optimal dose-per-fraction for high dose rate cervix cancer brachytherapy? Int J Radiat Oncol Biol Phys 1995;31:1011-3.

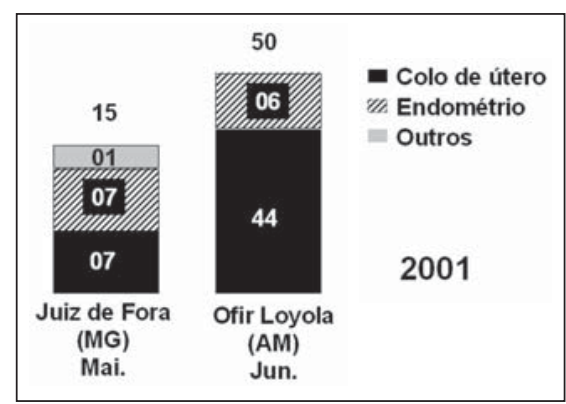

Gráfico 11. Número de pacientes tratados e distribuição das neoplasias em seus respectivos serviços (inaugurados em 2001).

10. Orton CG. High-dose-rate brachytherapy may be radiobiologically superior to low-dose rate due to slow repair of late-responding normal tissue cells. Int J Radiat Oncol Biol Phys 2001;49:183-9.

11. Arai T, Nakano T, Morita S, Sakashita K, Nakamura YK, Fukunisa K. High-dose rate remote afterloading intracavitary radiation therapy for cancer of the uterine cervix: a 20-year experience. Cancer 1992; 69:175-80.

12. Martinez A, Gonzalez J, Stromberg J, et al. Conformal prostatic brachytherapy: initial experience of a phase I/II dose-scalating trial. Int J Radiat Oncol Biol Phys 1995;35:1019-27.

13. Aisen S, Carvalho HA, Esteves SCB. High-dose rate brachytherapy in the treatment of carcinoma of uterine cervix. IX Congreso del Circulo de Radioterapeutas Ibero-Latinoamericano, Buenos Aires, Argentina, 10 a 13 de abril de 1994.

14. Aisen S, Carvalho HA, Petitto JW, Nadalin W, Haddad CMK. High-dose rate brachytherapy (HDRB) in the treatment of carcinoma of the uterine cervix - Brazilian experience. Int J Radiat Oncol Biol Phys 1998;421(Suppl):313 (abstract 2171).

15. Ferrigno R, Novaes PES, Pellizzon AC, et al. Highdose-rate brachytherapy in the treatment of uterine cervix cancer. Analysis of dose effectiveness and late complications. Int J Radiat Oncol Biol Phys 2001;50:1123-35.

16. Macha HN, Wahlers B, Reichle C, von Zwehl D. Endobronchial radiation therapy for obstructing malignancies: ten years' experience with iridium192 high-dose radiation brachytherapy afterloading technique in 365 patients. Lung 1995;173:271-80.

17. Perol M, Caliandro R, Pommier P, et al. Curative irradiation of limited endobronchial carcinomas with high-dose rate brachytherapy. Results of a pilot study. Chest 1997;111:1417-23. 